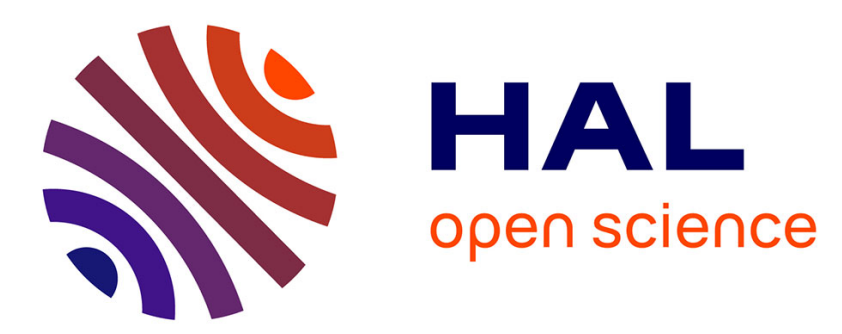

\title{
Appareillage pour l'étude en spectroscopie infrarouge de solides moléculaires sous haute pression (12 kbars) et a très basses températures $\left(4,2^{\circ} \mathrm{K}\right.$ et $\left.2^{\circ} \mathrm{K}\right)$
}

M. Jean-Louis, H. Vu

\section{- To cite this version:}

M. Jean-Louis, H. Vu. Appareillage pour l'étude en spectroscopie infrarouge de solides moléculaires sous haute pression (12 kbars) et a très basses températures $\left(4,2^{\circ} \mathrm{K}\right.$ et $\left.2{ }^{\circ} \mathrm{K}\right)$. Revue de Physique Appliquée, 1972, 7 (2), pp.89-94. 10.1051/rphysap:019720070208900 . jpa-00243610

HAL Id: jpa-00243610

https://hal.science/jpa-00243610

Submitted on 1 Jan 1972

HAL is a multi-disciplinary open access archive for the deposit and dissemination of scientific research documents, whether they are published or not. The documents may come from teaching and research institutions in France or abroad, or from public or private research centers.
L'archive ouverte pluridisciplinaire HAL, est destinée au dépôt et à la diffusion de documents scientifiques de niveau recherche, publiés ou non, émanant des établissements d'enseignement et de recherche français ou étrangers, des laboratoires publics ou privés. 


\title{
APPAREILlage POUR L'ÉTUde EN SPECTROSCOPIE INFRAROUGE DE SOLIDES MOLÉCULAIRES SOUS HAUTE PRESSION (12 kbars) ET A TRÈS BASSES TEMPÉRATURES $\left(4,2{ }^{\circ} \mathrm{K}\right.$ ET $\left.2{ }^{\circ} \mathrm{K}\right)$
}

\author{
M. JEAN-LOUIS et H. VU \\ Laboratoire des Hautes Pressions, CNRS, 92-Bellevue
}

(Reçu le 14 octobre 1971, révisé le 4 février 1972)

\begin{abstract}
Résumé. - On décrit un appareillage destiné aux études spectroscopiques de gaz solidifiés sous des pressions allant jusqu'à $12 \mathrm{kbars}$ et des températures pouvant atteindre $2{ }^{\circ} \mathrm{K}$. Le système se compose d'un cryostat optique à hélium liquide, d'une cellule optique et d'une presse capable de développer huit tonnes. Celle-ci actionne, par l'intermédiaire de deux longs tubes concentriques en acier inoxydable, un piston coulissant dans la cellule optique immergée dans l'hélium liquide. La pression est déterminée à $4,2^{\circ} \mathrm{K}$ par déplacement du flanc d'absorption d'un échantillon de $\mathrm{GaSb}$ inclus dans de l'hydrogène solide. Avec cet appareil, on a étudié l'évolution en fonction de la pression du spectre d'absorption induit dans le proche infrarouge de l'hydrogène solide pur.
\end{abstract}

Abstract. - An apparatus including a liquid helium cryostat, an eight ton press and a high pressure cell for $12 \mathrm{kbars}$ and $2{ }^{\circ} \mathrm{K}$ is described. Pressure measurements are made at $4.2^{\circ} \mathrm{K}$ by the displacement of the absorption edge of a GaSb sample imbedded in solid hydrogen. Some results on the fundamental vibration-rotation spectrum of pure solid hydrogen under pressure are given.

Une approche intéressante du problème des forces intermoléculaires intervenant dans les solides moléculaires consiste dans l'étude de l'influence exercée par la pression sur leurs spectres d'absorption infrarouge. Afin de pouvoir exploiter au mieux sur le plan théorique les résultats expérimentaux obtenus, on a intérêt à étudier des solides constitués de molécules très simples, telles que $\mathrm{H}_{2}, \mathrm{D}_{2}, \mathrm{~N}_{2}$, etc... Ces corps ont tous des points de solidification très bas et sont gazeux à la température ambiante, aussi est-on amené, en particulier dans le cas de $\mathrm{H}_{2}$ et $\mathrm{D}_{2}$, à utiliser un appareillage haute pression fonctionnant à la température de l'hélium liquide. Si cependant, comme dans le cas de $\mathrm{N}_{2}$, le point de solidification se situe moins bas, le fait de pouvoir travailler à cette température permet l'observation de changements de phase et une amélioration de la finesse des raies qui constituent les spectres. Enfin, les spectres d'absorption des corps mentionnés précédemment sont des spectres induits de faible intensité et il n'est possible de les observer que sous une épaisseur importante, excluant toute technique de dépôt sur fenêtre froide. L'appareillage haute pression et très basse température, dont nous donnons ici la description, a été conçu suivant ces critères.

Cet appareillage fait appel aux mêmes principes que ceux employés pour l'étude des équations d'état des solides moléculaires à très basses températures [1], [2]. Il se ícompose essentiellement [(Fig. 1) d'une presse à huile $(\mathrm{m})$ travaillant à la température ambiante, et qui exerce la force qu'elle développe, sur un piston (a) coulissant dans la cellule optique (q) immergée dans l'hélium liquide, par l'intermédiaire de deux longs cylindres concentriques (f) en acier inoxydable. Des fenêtres de saphir permettent l'observation spectroscopique dans le domaine du proche infrarouge suivant un axe perpendiculaire au déplacement du piston. Le solide à étudier, obtenu transparent sous forte épaisseur par solidification sous pression moyenne [3], est comprimé à haute pression à l'aide du piston seulement lorsque la cellule est à très basse température. Ceci permet de profiter de l'amélioration de la résistance mécanique du matériau de la cellule. De plus, les problèmes d'étanchéité de la cellule sous des pressions élevées ne concernent que la phase solide et sont, de ce fait, malgré la plasticité du solide, moins difficiles à résoudre que ceux posés par la phase gazeuse aux mêmes pressions.

Le cryostat à hélium liquide, de type classique, est en acier inoxydable. Il présente la particularité d'avoir toute sa partie inférieure complètement démontable, ce qui permet le montage d'appareillages volumineux de dimensions supérieures au diamètre du col du cryostat. L'étanchéité de l'enceinte intérieure contenant l'hélium liquide est assurée à l'aide d'un joint mince en téflon fortement serré entre deux brides, dont l'une porte un léger picot. Deux hublots de saphir démontables (p), diamétralement montés sur 


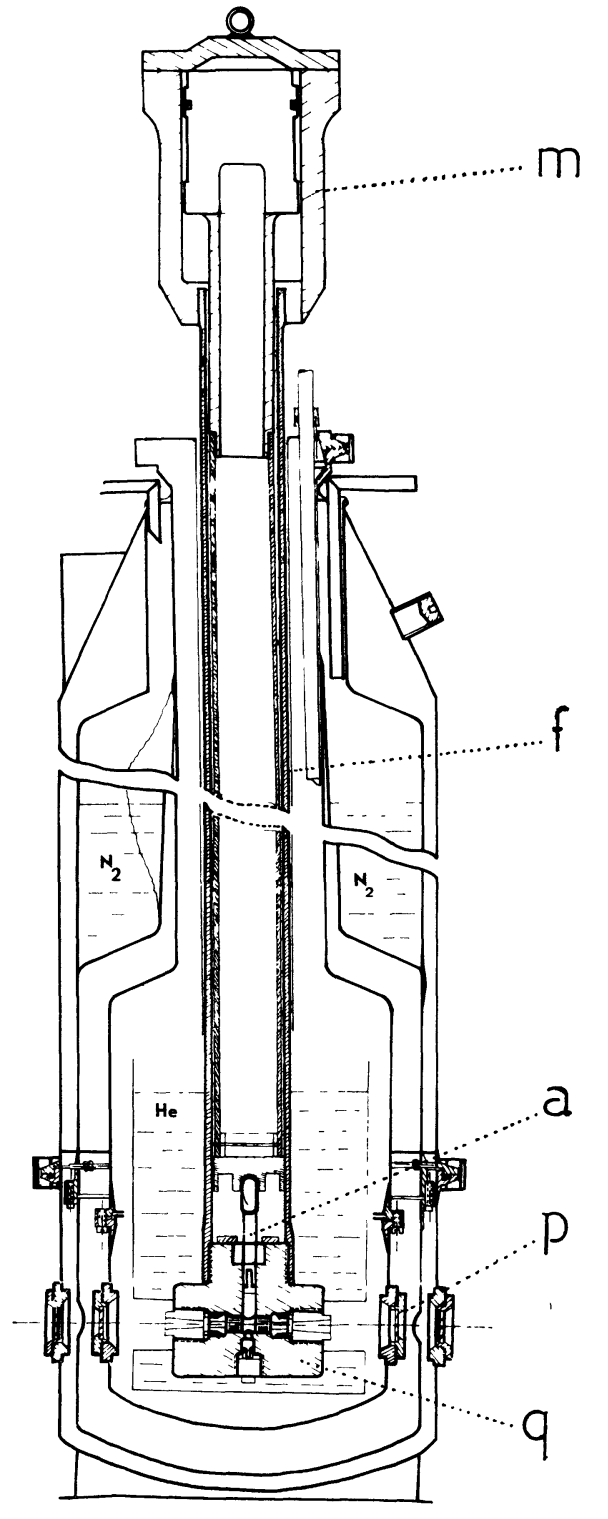

FIG. 1. - Schéma de l'appareillage.

la partie amovible de cette enceinte, permettent le passage du rayonnement infrarouge. Ils sont parfaitement étanches au vide, même lorsque l'une de leurs faces est en contact avec l'hélium liquide. Un vide primaire étant initialement réalisé entre les enceintes du cryostat, du charbon actif placé contre les enceintes à azote liquide et à hélium liquide assure un pompage cryogénique lors du refroidissement et évite l'emploi d'une pompe secondaire. La presse à huile est conventionnelle. Les bras de presse transmettant l'effort au piston sont deux longs cylindres concentriques de $80 \mathrm{~cm}$ de long, de $3,4 \mathrm{~mm}$ d'épaisseur et de diamètre respectif $52 \mathrm{~mm}$ et $38 \mathrm{~mm}$. Ils sont réalisés en acier inoxydable choisi pour sa faible conductivité thermique et permettent de transmettre une force de huit tonnes. Le guidage du tube de compression est assuré par plusieurs bagues, petites et ajustées, qui prennent appui sur la paroi intérieure soigneusement usinée du tube de tension. L'espace compris entre les deux tubes est maintenu étanche vis-à-vis de l'extérieur afin d'éviter tout risque de givrage. Le système haute pression peut être éventuellement raccordé au cryostat de façon étanche au niveau du tube de tension, si bien qu'il est possible de porter la cellule à une température de $2^{\circ} \mathrm{K}$ par pompage sur l'hélium liquide.

La cellule optique haute pression (Fig. 2) est réalisée en Berylco 25 testé aux ultrasons et traité àj dureté maximale. Elle présente une chambre cylindrique verticale dans laquelle peut coulisser la tête du piston. Celle-ci est constituée d'un ensemble de deux contrepistons (c) enserrant un joint de potassium (d) monté en aire non supportée et assure l'étanchéité de la

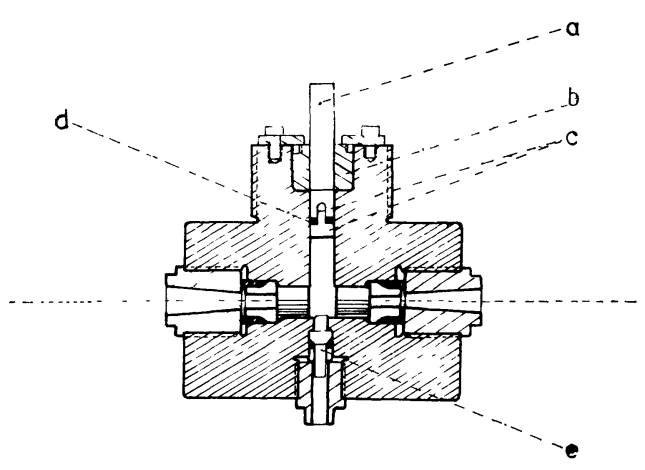

Fig. 2. - Schéma de la cellule optique.

partie supérieure de la cellule durant toute l'expérience. Le piston (a) poussé par le tube de compression de la presse coulisse à travers un canon de guidage (b) et déplace devant lui les deux contre-pistons (c) lors de la compression du solide. L'ensemble piston et contre-piston a un diamètre de $8 \mathrm{~mm}$, et est réalisé en acier indéformable traité à Rockwell C 60 . La paroi de la chambre cylindrique et les contre-pistons sont soigneusement usinés et ajustés, sans toutefois que l'ajustage puisse entrainer un grippage par dilatation différentielle. A la partie supérieure de la chambre, immédiatement sous le contre-piston en position haute, se trouve une arrivée de gaz (non représentée sur la figure 2), permettant le remplissage de la cellule. Cette arrivée de gaz est alimentée par un tube haute pression relié à l'extérieur et dont la partie froide est entourée d'une résistance chauffante ; l'ensemble tube-résistance étant lui-même entouré de fils d'amiante. Un tel dispositif réduit de façon très efficace le risque de formation de bouchons de solide à l'intérieur du tube lors de la condensation du gaz dans la cellule. La chambre se termine en bas par un orifice (e) pouvant s'obturer et permettant un serrage préalable du joint de potassium lors du montage de la cellule. L'intérêt de ce préserrage est de permettre d'obtenir dès le début de l'expérience une parfaite étanchéité : ce procédé ayant donné d'excellents résultats en toutes circonstances rend inutile le canon prévu pour porter un joint et a permis son élimination sur les versions plus récentes de la cellule. L'axe 
optique de la cellule est perpendiculaire au déplacement du piston. Les deux fenêtres sont constituées de cylindres de saphir de $10 \mathrm{~mm}$ d'épaisseur et de $10 \mathrm{~mm}$ de diamètre ayant un poli optique sur les deux faces. Elles sont portées par des embases réalisées avec le même acier que le piston. Ces dernières sont montées suivant le principe des joints « champignon » autoserreurs et font appel à des joints constitués d'empilement d'indium, de rondelles de cuivre recuit et de bronze au béryllium. En cours d'expérience, la cellule est immergée en permanence dans l'hélium liquide de façon que sa température soit fixe et uniforme. Aussi, pour ne pas être gêné au point de vue spectroscopique par les bulles résultant de l'ébullition de l'hélium liquide à $4,2^{\circ} \mathrm{K}$, le compartiment à hélium liquide est double, l'hélium liquide étant contenu dans le compartiment interne. Celui-ci est un simple récipient en tôle très mince raccordé de façon étanche à la cellule et conçu de façon à ce que le rayonnement infrarouge ne chemine pas à travers le liquide réfrigérant, bien que ce dernier entoure complètement la cellule.

Pour les expériences se déroulant à $4,2^{\circ} \mathrm{K}$, l'hélium gazeux provenant de l'évaporation du liquide est astreint, avant d'être évacué du cryostat, à circuler entre le tube extérieur de la presse et une mince jupe en acier inoxydable l'enserrant de très près. L'échange thermique forcé entre le gaz initialement très froid et le tube réduit les pertes introduites par celui-ci. D'autre part, l'appareillage est disposé entre le spectromètre (Perkin-Elmer 112 G) et le détecteur pour que le rayonnement qui traverse le cryostat soit monochromatique. Avec toutes les dispositions prises pour limiter les pertes thermiques, la consommation d'hélium liquide est acceptable. En effet, après avoir pré-refroidi le système avec de l'azote liquide, il est possible d'obtenir des solides transparents à $4,2^{\circ} \mathrm{K}$ et de les observer spectroscopiquement à diverses pressions pendant plus de quinze heures consécutives avec vingt litres d'hélium liquide.

Les expériences sont réalisées de la façon suivante : les corps à étudier $\left(\mathrm{H}_{2}, \mathrm{D}_{2}, \mathrm{Ne}, \ldots\right)$ étant gazeux à une température assez basse, on refroidit le système avec de l'azote liquide en maintenant dans la cellule une pression de l'ordre de $1 \mathrm{kbar}$ de gaz à étudier. Lorsque la température atteint environ $100^{\circ} \mathrm{K}$, deux cas peuvent se présenter quant à la solidification. Pour les corps à points de fusion bas, tels que $\mathbf{H}_{2}$, $\mathrm{D}_{2}, \mathrm{He}, \mathrm{Ne}, \ldots$, il suffit de transférer l'hélium liquide dans le cryostat en maintenant une pression comprise entre 1,1 kbar et 1,7 kbar dans la cellule : lorsque celle-ci se trouve portée à $4,2 \mathrm{oK}$, la solidification est achevée et l'on a alors de l'hydrogène solide, du deutérium solide, etc... parfaitement transparents sous une épaisseur pouvant atteindre $1 \mathrm{~cm}$. Pour les corps à points de solidification plus élevés, tels que $\mathrm{N}_{2}$, Ar, ..., on refroidit la cellule en maintenant une pression de 1,7 kbar jusqu'à une température d'environ $70^{\circ} \mathrm{K}$. La solidification est alors achevée et l'on maintient cette pression et cette température pendant environ deux heures durant lesquelles la transparence du solide s'améliore. Au bout de ce temps, et toujours à cette température, on comprime alors directement le solide à l'aide du piston jusqu'à une pression de l'ordre de 2,0 kbars et l'on attend encore deux heures de manière à obtenir une parfaite transparence. On porte ensuite la cellule à $4,2{ }^{\circ} \mathrm{K}$ en achevant le transfert d'hélium liquide, tout en maintenant en permanence durant tout le refroidissement le solide comprimé à environ 2,0 kbars par le piston. Cette technique de refroidissement d'un solide précontraint permet d'obtenir de l'argon (dopé en molécules $\mathrm{H}_{2}$ par exemple) ou de l'azote solide parfaitement transparent sous une épaisseur de $1 \mathrm{~cm}$ à la température de $4,2 \circ$. Cette technique de solidification est indispensable dans le cas de ces corps. En effet, si l'on refroidit ces solides de $70^{\circ} \mathrm{K}$ à $4,2^{\circ} \mathrm{K}$ sans les comprimer avec le piston, l'expérience montre que leur transparence se dégrade au cours du refroidissement et qu'ils deviennent rapidement opaques. On peut penser que cette dégradation provient de fractures apparaissant dans le solide par suite de la contraction thermique.

La cellule immergée en permanence dans le liquide réfrigérant se trouve portée à la même température que celui-ci. Une jauge électrique de niveau d'hélium liquide permet de vérifier à chaque instant que cette immersion est bien réalisée. De plus, le refroidissement est suivi à l'aide d'un thermocouple AuCo, puis d'une résistance au carbone. Pour les températures inférieures à $4,2^{\circ} \mathrm{K}$, la température est déterminée par mesure de la tension de vapeur du bain à l'équilibre thermique. L'épaisseur maximum d'absorption de la cellule est de $1 \mathrm{~cm}$, mais elle peut être réduite jusqu'à $1 \mathrm{~mm}$ par emploi de cales de saphir.

La détermination de la pression in situ a été réalisée par voie optique à l'aide d'un échantillon d'antimoniure de gallium en mettant à profit la plasticité de l'hydrogène solide à $4,2{ }^{\circ} \mathrm{K}$ [1]-[4]. Pour cela, on dispose une plaquette de GaSb parallèlement aux faces des fenêtres, de manière que tout le rayonnement infrarouge qui pénètre dans la cellule, traverse le semi-conducteur. Celui-ci, inclus dans de l'hydrogène solide dont la plasticité en fait un bon milieu transmetteur de pression à ces températures, se trouve ainsi porté à une température constante de $4,2^{\circ} \mathrm{K}$ pendant toute la durée de l'expérience. L'absorption fondamentale du $\mathrm{GaSb}$ se situant à une fréquence supérieure à celle de la région du fondamental de vibration rotation de $\mathrm{H}_{2}$, il est ainsi possible d'obtenir pour une pression donnée exercée par le piston sur le solide, à la fois le spectre de l'hydrogène et le flanc d'absorption de l'antimoniure de gallium. On peut ainsi relier l'évolution du spectre de l'hydrogène solide au déplacement du flanc d'absorption du semiconducteur. Connaissant ensuite le coefficient de pression du $\mathrm{GaSb}$ à température constante, cette évolution peut être reliée directement à la pression 
qui règne effectivement dans la cellule. Le coefficient de pression de l'échantillon de GaSb précédemment utilisé a été déterminé, dans une autre série d'expériences avec la même cellule aux températures de $77^{\circ} \mathrm{K}$ et $20^{\circ} \mathrm{K}$, entre $0,2 \mathrm{kbar}$ et $1,4 \mathrm{kbar}$. Le milieu transmetteur de pression utilisé est alors l'hélium qui est encore gazeux à $1,4 \mathrm{kbar}$ et $20^{\circ} \mathrm{K}$. Les valeurs trouvées sont $14,6 \mathrm{meV} / \mathrm{kbar}$ et $14,4 \mathrm{MeV} / \mathrm{kbar}$ respectivement à $77^{\circ} \mathrm{K}$ et $20^{\circ} \mathrm{K}$. Les valeurs s'écartent de celle déjà ancienne trouvée à l'ambiante par Drickamer [5]-[6] soit $12,3 \mathrm{MeV} / \mathrm{kbar}$, mais sont en bon accord avec la valeur de $14,5 \mathrm{MeV} / \mathrm{kbar}$, toujours à l'ambiante, trouvée plus récemment par Kosicki et Paul [7]-[8], par exemple. La mesure à $77^{\circ} \mathrm{K}$ étant moins précise que celle effectuée à $20^{\circ} \mathrm{K}$, il ne s'avère pas qu'il y ait de variations importantes du coefficient de pression entre ces deux températures. Aussi, la valeur utilisée pour déterminer la pression in situ dans le cas de l'hydrogène solide à $4,2^{\circ} \mathrm{K}$ a-t-elle été celle trouvée à $20^{\circ} \mathrm{K}$.

La pression maximale atteinte avec l'appareillage actuel est de 12 kbars réels à la température de $2^{\circ} \mathrm{K}$ obtenue sur de l'hydrogène solide. Les résultats dans ces dernières conditions seront publiés ultérieurement. Cette pression limite est fixée par des questions de résistance de matériau de la cellule et d'équation d'état du solide comprimé (limite imposée par la course maximum du piston). Une version améliorée de cet appareil faisant appel à un matériau plus résistant et une course de piston plus grande doit permettre de monter à une pression plus élevée dans un proche avenir.

Avec cet appareil, nous avons étudié l'évolution en fonction de la pression du spectre d'absorption induit dans le proche infrarouge de l'hydrogène solide normal à $4,2^{\circ} \mathrm{K}$ (Fig. 3). L'hydrogène solide est en effet particulièrement intéressant à étudier, car les molécules peuvent tourner quasi librement dans la phase solide par suite de la faiblesse des forces intermoléculaires. De plus, les masses des molécules étant petites, les raies de rotation sont parfaitement séparées. A faible pression $(0,7 \mathrm{kbar})$, le spectre obtenu (Fig. 3) est très voisin de celui observé par Welsh en l'absence de pression [9]. Il se compose de trois transitions simples de vibration-rotation $\left(\mathrm{Q}_{1}(\mathrm{~J})\right.$, $S_{1}(0)$ et $\left.S_{1}(1)\right)$ et de transitions doubles $\left(Q_{1}(J)+S_{0}(J), \ldots\right)$ résultant d'une combinaison entre une transition de vibration-rotation et une transition rotationnelle exécutées simultanément par deux molécules se partageant l'énergie d'un même photon. Chaque transition simple se compose d'une raie centrale (notée $\mathrm{Q}_{1 \mathrm{q}}(\mathrm{J}), \mathrm{S}_{1 \mathrm{q}}(0), \ldots$ ) correspondant à la transition de vibration-rotation proprement dite et de branches satellites (structures de phonons notées $\left.Q_{R}(J), S_{R}(0), \ldots\right)$ résultant d'une combinaison entre la fréquence de vibration-rotation et celles du réseau. D'une façon générale, l'évolution du spectre en fonction de la pression se caractérise par un déplacement général de l'ensemble des raies vers les hautes

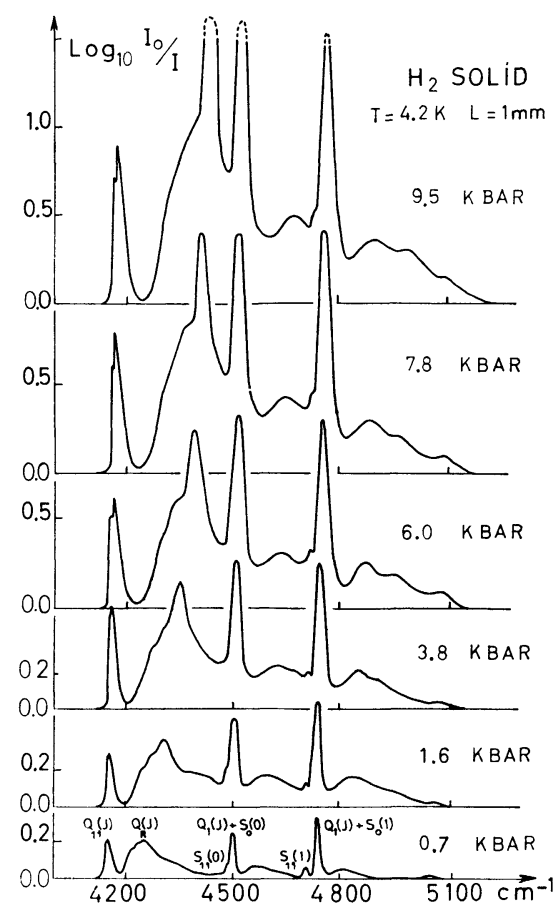

FIG. 3. - Evolution en fonction de la pression du spectre de l'hydrogène solide normal à la température de 4,2 o $\mathrm{K}$.

fréquences et une forte augmentation de l'intensité d'absorption. Dans le cas de transitions simples, il n'est possible de suivre que l'évolution de $Q_{1 q}(J)$ et $S_{1 q}(1)$ en fonction de la pression, car $S_{1 q}(0)$ disparaît très rapidement par suite de l'élargissement de la bande de transition simultanée voisine. L'évolution en fonction de la pression de la branche $Q_{1 q}(J)$ (composée en fait de $\mathrm{Q}_{1 \mathrm{q}}(0)$ et $\mathrm{Q}_{1 \mathrm{q}}(1)$ correspondant respectivement aux molécules d'hydrogène para et ortho) est donnée sur la figure 4. Dans la limite des erreurs expérimentales, le déplacement est linéaire (Fig. $4 b$ ) et a une valeur d'environ $2,5 \mathrm{~cm}^{-1} / \mathrm{kbar}$. En même temps qu'elle se déplace vers les hautes fréquences, la branche $\mathrm{Q}_{1 \mathrm{q}}(\mathrm{J})$ voit son intensité d'absorption intégrée multipliée par un facteur d'environ 7 entre $0,7 \mathrm{kbar}$ et $9,5 \mathrm{kbars}$, tandis que sa dissymétrie du côté des hautes fréquences est fortement accentuée (Fig. 4a). Un abaissement de la température jusqu'à $2 \circ \mathrm{K}$ montre que ce renforcement de la dissymétrie correspond à l'induction d'une bande large située à quelques $\mathrm{cm}^{-1}$ des raies fines $Q_{1 \mathrm{q}}(0)$ et $\mathrm{Q}_{1 \mathrm{q}}(1)$ [10]. Dans le cas de la bande $\mathrm{S}_{1 \mathrm{q}}(1)$ dans la transition $S_{1}(1)$, le déplacement est également linéaire, mais la proximité d'une bande de transitions simultanées ne permet pas de suivre l'évolution de son intensité d'absorption. Les bandes de transitions simultanées présentent elles aussi une augmentation importante de l'intensité d'absorption et leurs déplacements vers les hautes fréquences est du même ordre de grandeur que pour les transitions simples. Toutefois, elles subissent un élargissement qui rend plus imprécise la détermination de leurs fréquences. Les 


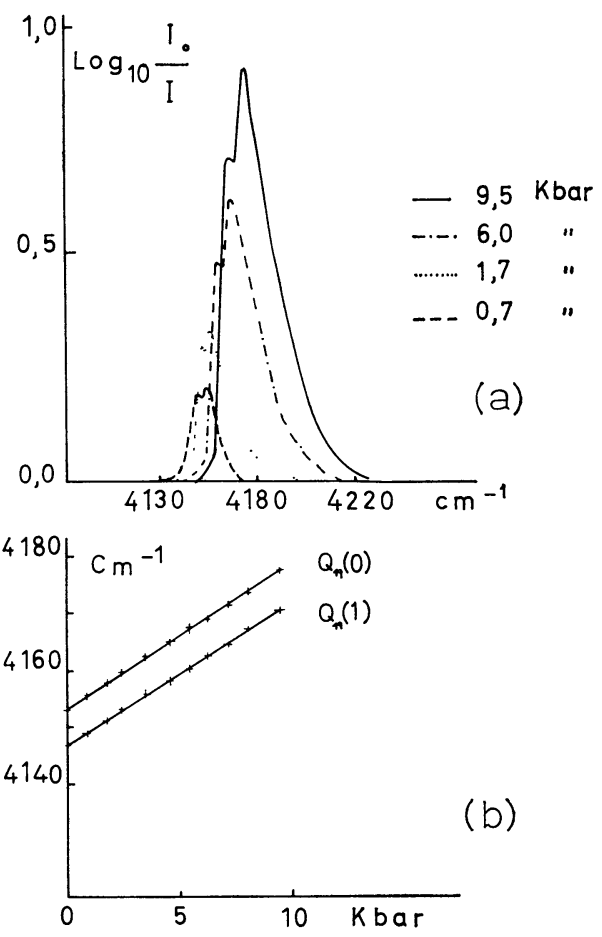

FIG. 4. - $a$ ) Evolution de $\mathrm{Q}_{1 \mathrm{q}}(\mathrm{J})$ en fonction de la pression.

b) Courbe représentant le déplacement de la fréquence de $\mathrm{Q}_{1 \mathrm{q}}(\mathrm{J})$ en fonction de la pression.

bandes de phonons présentent le même genre d'évolution, mais avec un caractère beaucoup plus accentué, en particulier dans le cas de $Q_{R}(J)$. En effet, tandis que son intensité intégrée est multipliée par un facteur d'environ 10 entre $0,7 \mathrm{kbar}$ et $9,5 \mathrm{kbars}$, on observe un fort changement de son profil. Le déplacement en fréquence du maximum est important, mais n'est pas une fonction linéaire de la pression. La relation entre pression et volume à $4,2 \circ \mathrm{K}$ étant connue dans le cas de l'hydrogène solide [2], il est intéressant de donner l'évolution du déplacement en fréquence de $Q_{1 q}(J)$ et de $Q_{R}(J)$ en fonction de la densité du solide, car cette grandeur est évidemment directement fonction du paramètre distance intermoléculaire. Les courbes sont données sur la figure 5. On constate que la courbe donnant le déplacement du sommet de $Q_{R}(J)$ en fonction de la densité devient sensiblement linéaire à haute densité, avec une pente d'environ $0,28 \mathrm{~cm}^{-1} /$ Amagat.

L'évolution de ce spectre en fonction de la pression est donc essentiellement caractérisée par un déplacement vers les hautes fréquences et une forte augmentation de l'intensité d'absorption de l'ensemble des bandes. Le déplacement vers les hautes fréquences s'explique qualitativement par la prédominance des forces de recouvrement sur les forces de dispersion. L'augmentation de l'intensité d'absorption peut s'expliquer par le fait qu'il s'agit de bandes induites très sensibles à la distance intermoléculaire. De plus, l'effet d'interférence [11] influe certainement beaucoup sur cette variation de l'intensité, car il est probable que, sous l'effet de la pression, il y ait modification de la symétrie de l'environnement de la molécule active.
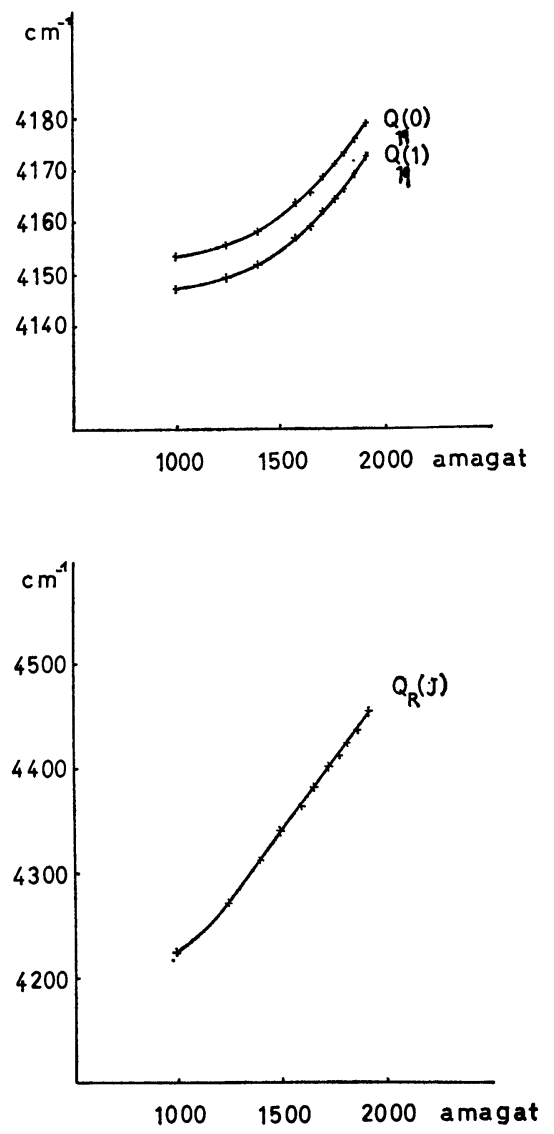

Fig. 5. - Courbes représentant l'évolution des fréquences de $\mathrm{Q}_{1 \mathrm{q}}(\mathrm{J})$ et $\mathrm{Q}_{\mathrm{R}}(\mathrm{J})$ en fonction de la densité.

En conclusion, on voit que cet appareillage permet l'étude spectroscopique dans le proche infrarouge sous hautes pressions et à très basses températures de corps à points de solidification très bas, tels que l'hydrogène, le deutérium, l'azote, etc... En outre, profitant des propriétés plastiques de corps tels que l'hélium solide, il est possible d'étendre son domaine d'utilisation à des études optiques de corps tels que les semi-conducteurs par exemple. Enfin l'appareil, moyennant des modifications mineures, peut se prêter à de multiples utilisations dans de nombreux autres domaines physiques, où sont nécessaires haute pression et basse température : études de diffusion Raman, étude des phénomènes électriques, etc...

Remerciements. - Nous tenons à remercier Messieurs Eymard et Ayrault, du CNET, de nous avoir fourni les échantillons de $\mathrm{GaSb}$ et conseillé à ce sujet.

Nous tenons également à remercier Monsieur Darpentigny, du Service des Prototypes du CNRS, pour l'aide apportée dans l'exécution des plans. 


\section{Bibliographie}

[1] Stewart (J. W.), Phys. Rev., 1955, 97, 578.

[2] Stewart (J. W.), J. Phys. Chem. Solids, 1956, 1, 146.

[3] Vu (H.), Atwood (M. R.), Staude (E.), C. R. Acad. Sci. Paris, 1963, 257B, 1771.

[4] Jennings (L. D.), Swenson (C. A.), Phys. Rev., 1958, $112,31$.

[5] Edwards (A. L.), Slykhouse (T. E.), Drickamer (H. G.), J. Phys. Chem. Sol., 1959, 11, 140.

[6] Edwards (A. L.), Drickamer (H. G.), Phys. Rev., 1961, 122, 1149.
[7] Kosicki (B. B.), Paul (W.), Phys. Rev. Letters, 1966, 17, 246.

[8] Kosicki (B. B.), Jayaraman (A.), Paul (W.), Phys. Rev., 1968, 172, 764.

[9] Gush (H. P.), Hare (W. F. J.), Allin (E. S.), Welsh (H. L.), Can. J. Phys., 1960, 38, 176.

[10] Jean-Louis (M.), Vu (H.), Données non publiées. [11] Van Kranendonk (J.), Physica, 1957, 23, 825. 